\title{
Editorial
}

\section{Tuberculous pericarditis and HIV infection}

Approximately 8 million new cases of tuberculosis were reported to the World Health Organization in 1997, and millions are infected with HIV and tuberculosis. ${ }^{1}$ Tuberculous (TB) pericarditis is increasing in sub-Saharan Africa, where tuberculosis is the most common opportunistic infection complicating HIV infection, and is seen occasionally in developed countries. ${ }^{2}$ It is likely to increase worldwide, because of poor tuberculosis and HIV control and the ease of travel from high to low risk areas. TB pericarditis is important because it is treatable and many patients can be managed wholly or predominantly as outpatients.

Management of TB pericardial effusion involves relieving tamponade if present, confirming and treating tuberculosis, preventing constriction if possible, and offering pericardiectomy if it persists. Correct management presupposes diagnosis, which is not a problem where it is endemic, ${ }^{3}$ but may be where it is uncommon. It is a reminder that diagnosing "congestive heart failure" is incomplete without a cause, and that pericardial effusion is confirmed par excellence by echocardiography. For many in sub-Saharan Africa, this will be done simply, using the general purpose or obstetrical ultrasound machine.

\section{The study of Hakim and colleagues}

It is fitting that an advance in managing TB pericarditis in HIV seropositive patients should be made in Zimbabwe, where Michael Gelfand emphasised its seriousness 50 years ago: "The prognosis in tuberculous pericarditis is invariably bad and death may take place within one or two years, but more often within a few months." " Since then, improved anti-TB treatment and benefits from adjunctive prednisolone in HIV seronegative patients ${ }^{56}$ have improved the prognosis. The study by Hakim and colleagues, in this issue, ${ }^{7}$ concerns the role of adjunctive prednisolone for six weeks in HIV seropositive patients with TB pericardial effusions receiving antituberculosis drugs for six months. In a randomised, placebo controlled, double blind study, the effect of prednisolone on resolution of pericardial effusions and mortality was investigated. Clinical and laboratory characteristics of the two groups were similar, apart from CD4 counts, which were higher in those who received prednisolone, although baseline counts were available from only 28 of the 58 patients. Pericardial effusions were confirmed by echocardiography.

Although all pericardial effusions contained a predominance of lymphocytes, culture grew Mycobacterium tuberculosis in only $38 \%$. Lowenstein-Jensen and double strength liquid Kirchner (DSK) were the culture media, but the numbers of each used and the drug sensitivity of the organisms are not mentioned. After six months, there was one death in the treatment group and nine in the control group. After 18 months, five had died in the treatment group and 10 in the control group.

In HIV seronegative patients with pericardial effusion, confirmation of the diagnosis of tuberculosis was considerably higher in two South African studies-that is, 58\% and $75 \% .{ }^{68}$ Confirmation of tuberculosis is achieved best by bedside inoculation of DSK, which is particularly appropriate for developing countries, being cheaper and better than more sophisticated techniques. There is insufficient experience with immunological, serological, and molecular biological techniques to recommend their routine use in developing countries; culture should be the standard by which the diagnosis is confirmed.

Although tuberculosis is the likely cause of pericardial effusion in HIV seropositive people in sub-Saharan Africa, other conditions may occur. Diagnostic pericardiocentesis is desirable, and in areas where amoebiasis is common, patients with pericardial effusion should have a liver ultrasound scan. ${ }^{9}$ HIV infected people are prone to pneumococcal infection and other infections and malignancies. When TB pericarditis is suspected, without evidence of another disorder, pericardial fluid should be examined by Gram stain and culture, Ziehl-Neelsen stain, differential cell count, and cytology. Before starting antituberculosis treatment blindly, the drug sensitivities of local organisms should be determined. This information is not always available in some settings. The pragmatic approach in Malawi, for example, is to treat pericardial effusions in HIV seropositive people as tuberculous. ${ }^{10}$ Where resources are better and tuberculosis is uncommon, more detailed investigation is essential, including, if necessary, pericardial biopsy for histology and culture.

\section{Benefits of adjunctive prednisolone}

Adjunctive prednisolone is beneficial in HIV seronegative patients with TB pericardial effusion, reducing the risk of recurrent tamponade, death from pericarditis, and constriction. ${ }^{6}$ Benefit from similar treatment in HIV seropositive patients is encouraging, particularly where the absence of antiretroviral drugs worsens the prognosis. Adjunctive prednisolone in Hakim's study resulted in significant improvement in physical activity and faster resolution of some physical signs of pericardial effusion in the treatment group, with a trend towards faster resolution of radiological and echocardiographic features.

Deaths were attributed to pericarditis or tuberculosis during the first six months and to various HIV related conditions thereafter, and were significantly less in the treatment group. Rifampicin, a hepatic enzyme inducer, causes increased metabolism of endogenous and exogenous corticosteroids, raising the possibility that some deaths in the control group were caused by Addison's disease, which can complicate HIV infection as well as tuberculosis and can be precipitated by rifampicin.

TB pericarditis has been described as a sign of disseminated disease in Zimbabwe, ${ }^{11}$ and $81 \%$ of Hakim's patients had generalised peripheral lymphadenopathy, although the extent to which tuberculosis was responsible is unknown.

\section{Future trends}

What of the future? For those doubting the benefits of prednisolone in TB pericarditis, ${ }^{12}$ further studies should be undertaken, although studies in HIV seronegative people may be increasingly difficult. The relatively small number of patients described by Hakim and colleagues requires further studies to confirm their findings, with attention to patient characteristics and comparability. Routine use of DSK should enhance the yield of tuberculosis and the possibility of alternative causes deserves attention. In those with impaired immunity, knowledge of drug sensitivities is important. Additional benefits are anticipated using a higher dose of prednisolone and a small study in South Africa supports this. ${ }^{13}$ Rapid clinical improvement occurred associated 
with reduction of cardiothoracic ratios, the greatest reduction occurring within the first week.

The results were particularly impressive, because most patients had tamponade on admission and none developed constriction. Such dramatic improvement justifies a larger dose of prednisolone initially, reducing it sooner and stopping it within 3-4 weeks. Tamponade is life threatening and many doctors are unwilling to undertake pericardiocentesis, so adjunctive treatment obviating it is welcome. The extent to which tamponade caused deaths in Hakim's control group is not mentioned.

Where antiretroviral drugs are available, interaction of protease inhibitors and rifampicin must be considered and guidelines for this have been issued by the Centers for Disease Control and Prevention. ${ }^{14}$

Coexistence of epidemic tuberculosis and HIV infection, interested clinicians, and good basic laboratory services in Zimbabwe and South Africa provide an appropriate environment for further work on TB pericarditis and adjunctive prednisolone.

Finally, the resurgence of all types of tuberculosis should alert general physicians and cardiologists in the developed world to the possibility of TB pericarditis. Awareness of tuberculosis should prompt appropriate microbiological investigation and initial management should include high dose prednisolone.

Colchester General Hospital

A WRAGG
Royal Glamorgan Hospital

J I G STRANG

Ynysmaerdy,

Llantrisant CF72 8XR, UK

1 Dye C, Scheele S, Dolin P, et al. Global burden of tuberculosis. FAMA 1999;282:677-86.

2 Williams IP, Hetzel MR. Tuberculous pericarditis in south-west London: an increasing problem. Thorax 1978;33:816-17.

3 Strang JIG. Tuberculous pericarditis. F Infect 1997;35:215-19.

4 Gelfand M. The sick African, 3rd ed. Cape Town:Juta and Co, 1957:494.

5 Strang JIG, Kakaza HHS, Gibson DG et al. Controlled trial of prednisolone as adjuvant in treatment of tuberculous constrictive pericarditis in Transkei. Lancet 1987; ii:1418-22.

6 Strang JIG, Kakaza HHS, Gibson DG, et al. Controlled clinical trial of complete open drainage and of prednisolone in treatment of tuberculous pericardial effusion in Transkei. Lancet 1988;ii:759-64.

7 Hakim JG, Ternouth I, Mushangi E, et al. Double blind randomized placebo-controlled trial of adjunctive prednisolone in the treatment of effusive tuberculous pericarditis in HIV sero-positive patients. Heart 2000;84: $183-8$.

8 Strang JIG, Latouf S, Commerford P, et al. Bedside culture to confirm tuberculous pericarditis. Lancet 1991;338:1601-2.

9 Strang G. Echocardiography in the developing world. In Wilde P, ed. Cardiac ultrasound. Edinburgh: Churchill Livingstone, 1993:289-303.

10 Maher D, Harries AD. Tuberculous pericardial effusion: a prospective clinical study in a low-resource setting-Blantyre, Malawi. International fournal of Tuberculosis and Lung Disease 1997;1:358-64.

11 Pozniak AL, Weinberg J, Mahari M, et al. Tuberculous pericardial effusion associated with HIV infection: a sign of disseminated disease. Tubercle Lung Dis 1994;75:297-300.

12 Mayosi BM, Volmink JA, Commerford PJ. Interventions for treating tuberculous pericarditis (Cochrane Review). In: The Cochrane Library, Issue 4, 1999. Oxford: Update Software

13 Strang JIG. Rapid resolution of tuberculous pericardial effusions with high dose prednisone and anti-tuberculous drugs. F Infect 1994;28:251-4.

14 Flexner C, Picitelli SC. Drug administration and interactions. In: Dolin R, Masur H, Saag MS. Aids therapy. New York: Churchill Livingstone 1999;785-97.

\section{IMAGES IN CARDIOLOGY}

\section{Giant right coronary artery fistula in a neonate}

Cardiac ultrasound (left) was performed on a 1 month old child with congestive heart failure. Both ventricles were dilated, and the right coronary artery was massively enlarged. There was torrential flow in the atrioventricular groove with the fistula entrance inferior and posterior to the septal leaflet of the tricuspid valve.

An ascending aortogram was performed in the lateral projection (middle). The posterior descending coronary artery (PDA) and posterolateral coronary artery (PL) arose at the origin of the fistula (RCA, right coronary artery; $\mathrm{RV}$, right ventricle). Test occlusions using a 5 French Berman balloon catheter identified a very limited segment where coil deployment would not cause inferior wall ischaemia. Given
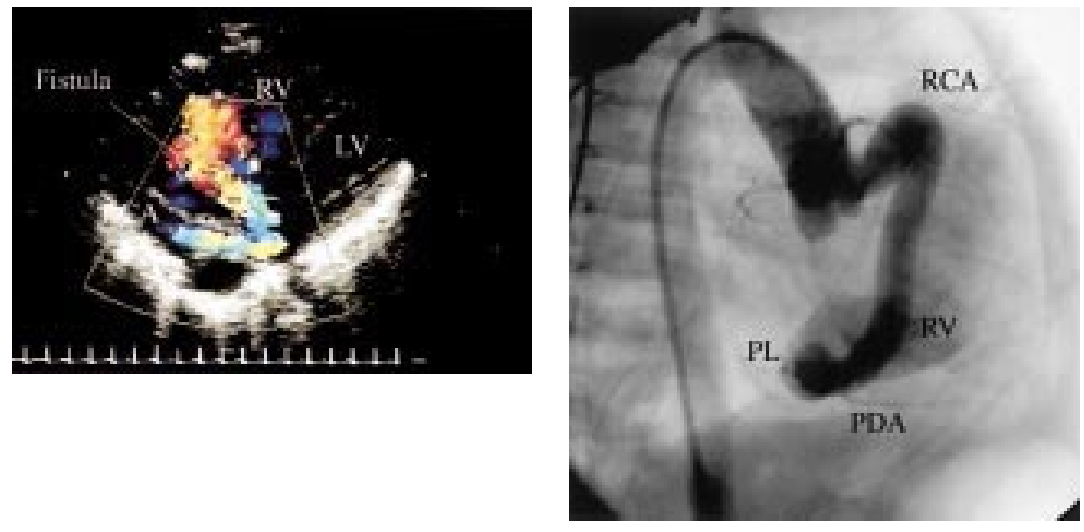

the small margin for error and the low operative mortality for this lesion, the patient was referred for surgery.

At operation a massively dilated right coronary artery was seen (right). The fistula insertion was posterior (not seen). The fistula's short, intramyocardial course precluded epicardial ligation so the fistula entrance into the right ventricle was oversewn through the tricuspid valve on the endocardial surface with pledgetted mattress sutures. The patient did well and was discharged eight days after the operation.

JOHN C WOOD GARY KOPF ALAN FRIEDMAN

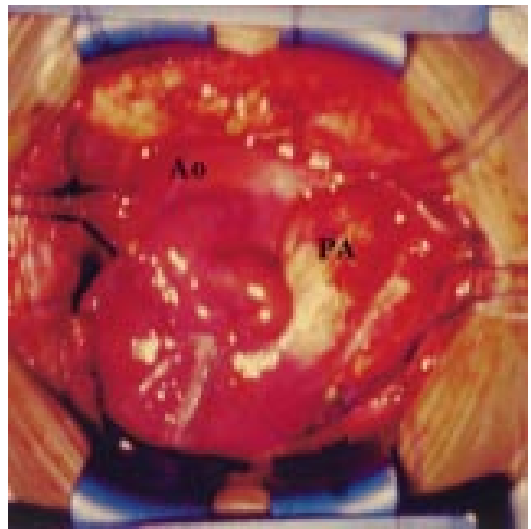

